\title{
The Influence of Language Transfer on Vocational Students' English Learning
}

\author{
Nuan Wang \\ Changchun Automobile Industry Institute, Jilin Province, China.
}

173265523@qq.com

Keywords: Vocational Students; Language Transfer; Second Language Acquisition

\begin{abstract}
Vocational students usually have poor basic skills in English. The reason is that most of the students lived in remote villages, counties, towns in China. The atmosphere of English learning in these areas is not as good as that in cities, and English teaching levels and teaching resources are limited there. When they speak English, their expressions are sometimes affected by Chinese seriously. The sentence structures of Chinese will appear in their English sentences frequently. This phenomenon, in the field of second language acquisition of linguistics, is called language transfer. Linguists have paid close attention to this learning state. There are two cases of language transfer: positive transfer and negative transfer. Most of vocational students are beginners of English and they are affected by Chinese transfer easily. Taking vocational students as the research object, this article studies and analyzes the influence of Chinese on English learning, hoping to help the students take advantage of the positive transfer, and avoid the negative transfer of Chinese on their English.
\end{abstract}

\section{Introduction}

As the first language, native language has influence on the second language acquisition. During vocational students' English learning, Chinese pronunciation, vocabulary and syntax have great influences on their English pronunciation, communication, and sentence patterns. Even their cultural knowledge affects all aspects of English learning. This phenomenon is known as language transfer in the study of second language acquisition. Odlin(2001), an American linguist, defines transfer as" the influence resulting from similarities and differences between the target language and any other language that has been previously acquired". Chinese transfer is also divided into positive and negative. The positive transfer is that there are similarities or consistencies of some pronunciations, sentence patterns, communicative situations, language rules, cultural customs and others between Chinese and English. Therefore, vocational students could learn English based on their Chinese knowledge. Because of the positive transfer, their English learning can be carried out smoothly. The positive transfer of Chinese language is active and stimulating. For vocational students, English teachers should make full use of the positive transfer of Chinese. The negative transfer of Chinese, by contrast, refers that some of Chinese pronunciation, grammars, communication contexts, language rules and cultural customs have differences with those of English, and students would like to apply the rules of Chinese to English, which led to mistakes in English. This is a very common phenomenon in students' English learning, and it is not easy to correct. In the teaching process, it is necessary for English teachers to pay close attention to Chinese negative transfer of the students, to rectify in time in order to avoid fossilization, which means the errors are difficult to correct. If fossilization occurs in the students' English, mistakes will last a lifetime, and the input of new knowledge and correct rules of English will be interrupted. The ultimate goal of language learning is to achieve communicative functions, while the negative transfer of Chinese can make obstacles to communication, and even pull a boner. In recent years, not only the linguists, but also English teachers begin to pay more and more attention to the negative transfer of mother tongue. English teachers in vocational schools are working hard to avoid the bad influence of the negative transfer of Chinese on students' English learning. This 
article summarizes the influence of the positive and negative transfers of Chinese on vocational students' English, and studies the learning disabilities caused by the negative transfer of Chinese. By doing the research, the author is willing to provide countermeasures for vocational schools' English teachers to overcome the influence of the negative transfer of Chinese.

\section{The Positive Transfer of Chinese}

As human communication tools, languages have certain similarities and common characteristics. With the deepening of globalization, the communication between countries is becoming more and more frequent. Foreign cultures have impacts on local culture and even integrated with native culture. Languages are the carriers of different cultures. Second language learning tends to begin with students' existing experience of their mother tongue. The common features of languages enable students to use some of the characteristics of Chinese on English learning. Vocational students' Chinese language structure is completed. Therefore, in their learning process, there are obvious signs of the positive transfer of Chinese on their English learning. Here are some examples.

\subsection{The Structure of the Declarative Sentences}

No matter English or Chinese has declarative sentences, and the word orders in both languages are with consistency. The basic structure of declarative sentences is: subject, predicate, and object. The simpler the structure and the tense of a declarative sentence are, the easier it is for vocational students to speak English correctly. For example, Chinese sentence: “我喜欢英语课。” English sentence: "I love English lessons." This example is the simplest declarative sentence only with subject, predicate and object. The tense of the English sentence is the present tense, which is exactly the same as the Chinese one. Another example: “我昨天上了一节英语课。” “I had an English class yesterday. " This sentence is a little more complicated. In this example, there will be a few vocational students who begin to make mistakes, but the mistakes are not about the word order. It is the past tense that most of the vocational students would make mistakes on. The reason is that English and Chinese have differences in the expression of the past tense. In Chinese, the speaker expresses the past tense by adding a Chinese character "了", but in English he needs to reflect the past tense by changing the verb from have to had; Another example: “我已经吃了蛋糕。” “I have eaten the cake." Vocational students would make many mistakes here. Because there are many changes in English perfect tense, such as adding auxiliary verb: have, changing the verb eat into its perfect tense eaten. Vocational students may be too careless to make a correct sentence. If the sentence is long and complicated, including attributive, adverbial, adverbial, etc., there will be more and more mistakes. To overcome this problem, the author suggests that in the teaching process, teachers make full use of positive transfer of Chinese, letting students make a simple declarative sentence with subject, predicate and object first, then complement the sentence with attributive, predicative, adverbial in the right place. After such training exercises, vocational students can make correct sentences that conform to English grammar slowly.

\subsection{The Pronunciation of Words}

With the integration of culture, more and more English words are pouring into Chinese. The pronunciation of such words in Chinese is very similar to that in English. Most of vocational students would not devote enough time to memorize words, but it takes them a very short time to memorize foreign words. For example: jeep (吉普), guitar (吉他), humor (幽默), show (秀), soda (苏打水), coffee (咖啡), chocolate (巧克力), Chicago (芝加哥), Harvard (哈佛), Columbia (哥伦比亚), etc.; and some onomatopoetic words, such as tick (滴答), hi (烸), Wow

(哇), ouch (哎呦), are easy to remember, because no matter Chinese or English, onomatopoetic words are created by imitating sounds. Teachers can make the comparison between Chinese and English so that students can remember them easily. 


\subsection{The Formation of Words or Phrases}

Some words or phrases in English have similar formation with Chinese words or phrases, and even the meanings are very similar. Take the word heart as an example. In English, there is a phrase: break one's heart, which means “伤心” in Chinese. The word break means “伤” in Chinese, and the word heart means “心”in Chinese. So the meanings of the two English words in this phrase are coherence with the two characters' Chinese meanings. There are many examples here, such as warm-hearted (热心肠), lose heart (灰心). The similar formation of words or phrases could be a kind of positive transfer of Chinese in English learning process. Such similarity makes it possible for vocational students to make use of the positive transfer of Chinese.

\section{The Negative Transfer of Chinese}

Chinese and English belong to different language families. Chinese, a kind of analytic language, is in Sino-Tibetan language family. One of the characteristics of Chinese is that it is a tone language, which means the grammar of Chinese is not implemented by changing the internal form of words, but by adding independent function words to reflect the changes of the grammar. However, English belongs to the Indo-European language family, and the Indo-European language family belongs to the fusional language, whose typical characteristic is that there is a change in person, number, tense, and voice. The base of English of vocational students is not firm. When vocational students speak English, two completely different language systems will appear in their minds at the same time, resulting in the confusion of languages. In addition, the students are particularly willing to use Chinese grammar rules to make English sentences because they already have more than ten years' thinking habits of Chinese, This has resulted in the negative transfer of Chinese, which hinders the students' interest in learning English seriously. Many students tend to lose their confidence in learning English because of the negative transfer of Chinese.

\subsection{The Impact of Pronunciation}

There are 21 initials and 19 finals in Chinese, but there are 20 vowels and 28 consonants in English. There are differences among pronunciation, rules and spellings. A Chinese Pinyin is composed of initials and finals, but an English word is a "vowel + consonant" structure, such as: egg/eg/; or a "consonants + vowels" structure, such as: pea/pi:/. Vocational students are often influenced by the pronunciation of Chinese, and they often add an extra vowel /ə/ to the consonant, such as the word : cool/ku:/, many students prefer to pronounce it like /ku:lə/. English has special phonetic symbols which do not exist in Chinese, such as $/ \theta / a n d / \delta /$. These two English phonetic symbols belong to the interdentals, but Chinese does not have such Pinyin. Students always read the word this like/zis/, the word think like /xink/. These examples prove that the pronunciations of English and Chinese are totally different. If Chinese pronunciation habits is transferred to English pronunciation, mistakes can be caused, which may leads to students' incorrect pronunciation.

\subsection{The Usage of Words}

The characters used to express something in Chinese are relatively fixed, but sometimes there are several words to express one thing in English. For example, let's talk about the Chinese character “吃”. In Chinese phrases: “吃饭”, “吃面条”, “吃鸡蛋”, we use the same character “吃” meaning eat. But in English, there are two words having the same meaning with the character “吃”: have and eat. For example, have breakfast, eat noodles and eat eggs. Besides, the adjective used in a phrase may be different. For example, in Chinese, we use the character “多”, which means many / much in English, to describe the population of a country. But in English, we say, "The population is large.” Students are confused because large means “大” rather than “多” in Chinese. Another obvious example is the character "开". In Chinese, we have the phrases: “开会”, “开车”, “开学”. Actually, “开” has different meanings in each phrase. But if the students used open to translate “开”, 
mistakes would be made.

\subsection{The Influence of Communication Context}

By their family background, education, living habits and cultural customs, vocational students' communication in English is often affected by the negative transfer of Chinese. For example, the use of the sentence: thank you. In English context, Thank you is the most common reply when being congratulated or praised. However, Chinese considers humility as a virtue and they believe that people should not accept others' praise directly. People often express their thanks by self-mockery. To Chinese people, the context should be answered in English like this, "You have overpraised me." or "I feel ashamed." Such an answer would make foreigners confused and even misunderstood. Another example, an English salesman would say "Can I help you?" to the customer. But a Chinese salesman would express the same meaning by saying "What would you like?" "What do you want?" in English. Firstly, this expression is not a correct expression in English. Secondly, if a salesman said the last two sentences, it would convey a feeling of impatience and reprimand. The phenomenon of the negative transfer of Chinese is very common in vocational students' English. If teachers want to avoid the negative transfer, they need to teach the comparison of Chinese culture and western culture in English classes, letting students understand the differences between Chinese and western cultures.

\section{Conclusion: The Impact of Language Transfer on English Teaching}

From the above argument, it can be seen that the negative transfer of Chinese can be embodied in the aspects of phonetics, vocabulary and context. If teachers want to avoid the effect of negative transfer, it is necessary to help students strengthen the basics of English and deepen the interpretation of vocabulary and grammar. What is more important is that Chinese and western cultures should be involved in the curriculum. Students in vocational colleges are not active in English learning. Some vocational students are influenced by their family environment, education atmosphere and other aspects, that's why their communicative ability is so weak. Therefore, teachers need to help students develop good language learning habits and help students develop their English thinking when teaching English. For the positive transfer of Chinese, teachers should make full use of it. For the negative transfer of Chinese, we should explore the reasons so as to overcome the students' difficulties in English learning.

\section{References}

[1] Chiang, Thomas. Some interferences of English intonation with Chinese tone. [J].International Review of Applied Linguistics,1979,(17):245-250.

[2] Ellis, Rod. The Study of Second Language Acquisition. Oxford University Press,1985.

[3] Selinker L. Interlanguage [J]. International Review of Applied Linguistics,1972,(10):209-230.

[4] Weidong Dai, Dong Wang. Language Transfer Research: Question and Thinking [J].Foreign Languages, 2002(6):93-100.

[5] Chengxian Tang. Language Negative Transfer in Second Language Acquisition[J]. Journal of PLA Foreign Languages Institute, 2003(5).

[6] Juan Yan. The Negative Transfer of Chinese and Coping Strategies in English Learning[J]. Technical Information (Academic Research),2007(5).

[7] Yuanyuan Li, Yang Zhao. The Language Transfer in Second Language Acquisition[J]. Journal of Hubei Radio and Television University, 2008.

[8] Jing Zheng. On Language Transfer in Foreign Language Learning[J]. Journal of Fujian Normal University Fuqing Branch,2004. 